group include chaperones, heat shock proteins and subunits of the $26 \mathrm{~S}$ proteasome which are all decisively involved in protein homeostasis. Another emerging GO group is translation. Crucial proteins such as the methyltransferase fibrillarin aggregate, segregate and thus reduce translational activity. The metabolic changes contribute to neurodegeneration and starvation which in turn manifests in petite worms showing motoric and olfactory deficits. Conclusions: A picture emerges that pollutants induce premature aging, thereby reducing the health span and longevity of $\mathrm{C}$. elegans. The neural system of the worm represents a prominent target organ showing neuromuscular defects that among others manifest in neurodegeneration of single neurons in combination with altered locomotion, reduced fecundity and impaired olfaction. As C. elegans leads a dual life, e.g. in the lab and in ecosystems, our results explain how environmental pollutants reduce the fitness of wild worms. Concerning the survival of wild populations the flexibility of adaptive gene expression and proteomes likely determines biodiversity, namely resilience against pollutantand climate-stress.

doi: http://dx.doi.org/10.7124/bc.0009CD

\section{Non-random chromosome segregation in Mesostoma ehrenbergii spermatocytes}

Eleni Fegaras and Arthur Forer

Biology Department, York University, Toronto, ON Canada M3J 1P3

efegaras@gmail.com
Mesostoma erhenbergii are hermaphroditic aquatic flatworms that reside in rivers and lakes across North America and elsewhere. Their primary spermatocytes have a very unusual meiosis (reviewed by Ferraro-Gideon et al. 2014). The cells have four univalent chromosomes, of two different kinds, that stay at the spindle poles, plus three bivalents. Some evidence suggests that the chromosomes are distributed via non-random segregation. For example, univalents move from pole to pole to obtain proper segregation, but they do this more often than necessary to obtain one of each kind at each pole, and often homologous chromosomes switch places with one another (Oakley 1984). Another example is that oriented bivalents sometimes detach and reorient to the opposite poles (Ferraro-Gideon et al 2014). Here we provide further evidence of non-random segregation in Mesostoma spermatocytes. Methods: Testes extracted from living Mesostoma ehrenbergii using a pulled glass needle are expelled onto a coverslip into Ringers solution that contained fibrinogen, and are held in a fibrin clot. Nocodazole (NOC) was perfused during prometaphase. We recorded live cells and studied the time-lapsed images. Results: NOC depolymerizes spindle microtubules. According to standard theories of mitosis, if microtubules are quickly depolymerized the chromosomes should arrest at the metaphase plate and not move. In Mesostoma spermatocytes, however, immediately following drug addition the chromosomes stretch out and, after a few minutes, in 52/59 cells the bivalent kinetochores detach from one pole and all move rapidly toward the other pole (Fegaras and Forer 2018 a and b). We have eliminated some other possibilities, and sug- 
gest that the non-random movement to one pole is indicative of normally occurring nonrandom segregation of chromosomes. Conclusions: There are two suggestive pieces of evidence that there is non-random segregation in Mesostoma spermatocytes. Our experiments add a third piece of evidence. We hope to test further for non-random segregation using micromanipulation techniques to move chromosomes around in the cell, and to mate our strain with European strains of the same species that have different chromosome configurations.

References: Fegaras E, Forer A. (2018a). Protoplasma 255: 1205-1224. Fegaras E, Forer A. (2018b). Protoplasma 255: 1401-1411. Ferraro-Gideon J, Hoang C, Forer A. (2014). Protoplasma 251: 127-143. Oakley, H A. (1984). Chromosoma 91: 95-100.

doi: http://dx.doi.org/10.7124/bc.0009CE

\section{Common and distinct roles of the isoforms of histone variant $\mathrm{H} 2 \mathrm{~A} . \mathrm{Z}$ in transcriptional regulation}

Daisuke Takahashi ${ }^{1}$, Noboru Ogihara ${ }^{1}$, Masayuki Kusakabe ${ }^{2}$, Saho Kitagawa ${ }^{1}$, Yukako Oma $^{1}$, Masahiko Harata ${ }^{1}$

${ }^{1}$ Graduate school of Agricultural Science, Tohoku University, Sendai, Japan; ${ }^{2}$ Biosignal Research Center, Kobe University, Kobe, Japan azuki.through@me.com

Nucleosomes consisting of DNA and core histones are the basic units of chromatin and play central roles in the regulation of genome functions. Histone variants are structurally similar to canonical histones, and the ex- change of canonical histones to their variants contributes to chromatin modulation and various epigenetic regulations. H2A.Z is an evolutionarily conserved $\mathrm{H} 2 \mathrm{~A}$ variant reportedly involved in the regulation of genome functions, including chromosomal segregation and transcriptional regulation. However, molecular mechanisms of H2A.Z functions have not been fully elucidated. In particular, molecular functions of two isoforms of vertebrate H2A.Z (H2A.Z.1 and H2A.Z.2) have not been analyzed specifically. On the other hand, when considering the biological phenomena, only H2A.Z.2 reportedly has a role in driving malignant melanoma (Vardabasso et al, Mol Cell, 59, 2015). To investigate molecular functions of the H2A.Z isoforms, we established gene knockout cells for H2A.Z.1- or H2A.Z.2-regulated genes with chicken DT40 cells (Z.1-KO or Z.2-KO cells, respectively). We found that some, but not all, genes are differently dysregulated in Z.1$\mathrm{KO}$ and Z.2-KO cells. Genes for transcriptional regulators, including cancer associated genes, are differently dysregulated in Z.1-KO and Z.2-KO cells, especially under oxidative stress conditions. Chromatin immunoprecipitation analysis revealed that H2A.Z.1 and H2A.Z. 2 have common and distinctive roles in the regulation of these genes. Our study showed that H2A.Z.1 and H2A.Z.2 have common and distinctive functions in epigenetic regulation.

Reference: Vardabasso, C., Gaspar-Maia, A., Hasson, D., Pünzeler, S., Valle-Garcia, D., Straub, T., ... Bernstein, E. (2015). Histone Variant H2A.Z.2 Mediates Proliferation and Drug Sensitivity of Malignant Melanoma. Molecular Cell, 59(1), 75-88. https://doi.org/10.1016/j.molcel.2015.05.009 